\title{
Influence of intensive multifunctional neuro- rehabilitation on neuronal oxidative damage in patients with Huntington's disease
}

\author{
Irene Ciancarelli, MDa,b \\ Daniela De Amicis, MSc ${ }^{a}$ \\ Caterina Di Massimo, MSc ${ }^{a}$ \\ Giorgio Sandrini, MDc \\ Caterina Pistarini, MD ${ }^{d}$ \\ Antonio Carolei, MDa \\ Maria Giuliana Tozzi Ciancarelli, MSc ${ }^{a}$
}

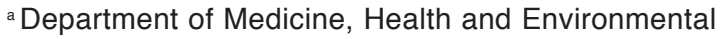

Sciences, University of L'Aquila, L'Aquila, Italy

${ }^{b}$ Nova Salus Rehabilitation and Nursing Home,

Trasacco (L'Aquila), Italy

${ }^{c}$ Neurorehabilitation Unit, C. Mondino National

Neurological Institute, Pavia, Italy

${ }^{d}$ Departments of Neurorehabilitation and Coma; Spinal Unit, Salvatore Maugeri Foundation, Pavia, Italy
\end{abstract}

Correspondence to: Irene Ciancarelli

E-mail: irene.ciancarelli@univaq.it

\section{Summary}

The influence of intensive multifunctional neurorehabilitation on serum levels of $\mathrm{Cu} / \mathrm{Zn}$-superoxide dismutase (Cu/Zn-SOD), neuron-specific enolase (NSE), and 8-hydroxy-2-deoxyguanosine (8-OHdG), as markers of oxidative damage, was evaluated in symptomatic patients with Huntington's disease (HD). Improved clinical outcome measures were observed after neurorehabilitation. Baseline levels of $\mathrm{Cu} / \mathrm{Zn}-\mathrm{SOD}$, NSE and 8-OHdG were higher than those observed in controls. $\mathrm{Cu} / \mathrm{Zn}$-SOD and NSE values decreased after neurorehabilitation, but were still higher than those measured in controls. $\mathrm{Cu} / \mathrm{Zn}-\mathrm{SOD}$ and NSE correlated positively before $(r=0.659 ; p=0.003)$ and after rehabilitation $(r=0.553$, $p=0.017) .8-O H d G$ values decreased after neurorehabilitation without reaching significance when compared with baseline values $(p=0.145)$. No correlation was observed between the measured oxidative markers and the assessed clinical outcome measures, either before or after neurorehabilitation. The findings reported in the present paper provide evidence of the effectiveness of neurorehabilitation in reducing oxidative damage in HD patients and underline the limit of serum oxidative markers for the evaluation of clinical features of HD.
KEY WORDS: CU/Zn-superoxide dismutase, Huntington's disease, neuron-specific enolase, neurorehabilitation, oxidative stress, 8hydroxy-2-deoxyguanosine.

\section{Introduction}

Huntington's disease (HD) is a fatal neurodegenerative disease caused by a cytosine-adenine-guanine repeat expansion of variable length in the huntingtin gene that translates into an abnormal polyglutamine repeat in the mutant huntingtin (mhtt) protein (Andrew et al., 1993; Aziz et al., 2009). The events caused by mhtt or its fragments lead to selective neurotoxicity and death of GABAergic striatal neurons and, in the long term, of cortical neurons (Eidelberg and Surmeier, 2011). As the disease progresses, the thalamus, hippocampus and amygdala are also involved and damaged (Armstrong and Miyasaki, 2012). Clinical manifestations in HD patients, which present as involuntary choreiform movements, psychiatric symptoms and cognitive impairment, result in progressive disability and lead to premature death (Aziz et al., 2009; Montoya et al. 2006). A complex gender effect on disease severity and rate of progression in $\mathrm{HD}$ has also recently been observed, with a more severe phenotype and faster rate of progression found in female HD patients (Zielonka et al., 2013). The cause of neurodegeneration and neuronal death in HD has not been completely identified, although many studies have shown aberrant interactions between mhtt and neuronal mitochondria that become dysfunctional and simultaneously a source of free radicals and a target of free radical attack (Perluigi et al., 2005; Sorolla et al., 2008). Free radicals generated in excess within dysfunctional mitochondria support a long-lasting oxidative condition that may promote the dysfunction and loss of neurons occurring in the brains of HD patients (Sorolla et al., 2008; Trushina and McMurray, 2007). Accumulation of oxidative by-products, specifically within the neostriatum, and a decreased antioxidant capacity confirm that oxidative stress is strongly involved in the neurodegeneration and neuronal death observed in HD patients (Johri and Beal, 2012; Milakovic and Johnson, 2005; Stack et al., 2008). Interestingly, it has been suggested that the oxidative damage through which mhtt leads to neuronal injury may be shared between central nervous system and periph- 
eral tissues (Sassone et al., 2009). Accordingly, elevated levels of oxidative by-products and decreased antioxidant defences were observed in serum/plasma or peripheral tissues of HD patients suggesting the potential significance of their evaluation, still to be validated (Borowsky et al., 2013; Ciancarelli et al., 2014), as promising peripheral markers useful for evaluating disease severity and for monitoring disease progression (Chen et al., 2007; Klepac et al., 2007; Hersch et al., 2006; Túnez et al., 2011). Recently, evidence emerged that moderate aerobic exercise may promote antioxidant capacity on the brain (Camiletti-Moirón et al., 2013) and that intensive neurorehabilitation, in spite of the predominantly static nature of most exercise protocols designed to improve muscle strength and coordination, enhances functional training and improves aerobic fitness, known to positively modulate the redox equilibrium (Al-Jarrah and Jamous, 2011; Arida et al., 2013; Ciancarelli at al., 2012; Tuon et al., 2012). Therefore, it could be hypothesized that physical exercise, included in an intensive and multifunctional neuromotor rehabilitation program as a specific strategy to lessen the patient's disability and delay the progression of motor dysfunction, could modulate the oxidative damage occurring in HD patients and, consequently, modify blood levels of oxidative by-products. The aim of this study was to investigate the potential effects of a three-week intensive, multifunctional neurorehabilitation program (designed to reduce functional impairments and to improve motor capacities of symptomatic HD patients) on serum levels of $\mathrm{Cu} / \mathrm{Zn}$ superoxide dismutase (Cu/Zn-SOD), neuron-specific enolase (NSE) and 8-hydroxy-2-deoxyguanosine (8-OHdG) as peripheral markers of the neuronal oxidative damage occurring in HD.

\section{Materials and methods}

Thirty-four patients with a clinical diagnosis of HD, confirmed by diagnostic genetic testing, undergoing in-hospital neurorehabilitation at the Nova Salus Rehabilitation Center (NSRC), were recruited from January 2011 to January 2012. A multidisciplinary team assessed independence and performances in activities of daily living (ADL) through administration of the Barthel Index (BI) (10-item) (Mahoney and Barthel, 1965) and the Total Functional Capacity Scale (TFCS) (5-item), which is a section of the Unified Huntington's Disease Rating Scale (Siesling et al., 1998; Shoulson, 1981). Motor performances on functional tasks were assessed using the Physical Performance Test (PPT) (9-item) (Reuben and Siu, 1990); balance and gait were evaluated using the Tinetti scale (TS) (16-item) (Tinetti, 1986). Included patients had to be free of other neurological and psychiatric diseases liable to interfere with an acceptable cognitive performance, defined as the ability to perform the exercises proposed by the physiotherapists, with a preserved autonomy in self-ambulation confirmed by a score $\geq 1$ on the 9th item of the PPT.
Twelve HD patients $(35.3 \%)$ under tetrabenazine treatment, two patients $(0.68 \%)$ receiving antioxidants and vitamin supplementation, and two patients $(0.68 \%)$ suffering from diabetes mellitus were excluded to avoid confounding factors known to influence oxidative stress markers. Eighteen patients with HD, 13 men and 5 women (mean age $51.11 \pm 2.77$ years), fulfilled the criteria and were thus included in the study. The patients' mean age at onset of symptoms was $31.29 \pm 7.40$ years (age range 27 to 35 years). Ten apparently healthy volunteers, four men and six women (mean age 50.00 $\$ 3.59$ years) recruited among outpatients referred to the NSRC for routine laboratory examinations, were enrolled as controls to compare oxidative markers. General lifestyle habits, including current daily consumption of antioxidants in foods and beverages, were carefully monitored by means of a structured questionnaire in all the participants. The study design, approved by the local ethics committee and performed in accordance with the Declaration of Helsinki, was explained in advance to all eligible participants or to their caregivers who gave their written informed consent.

\section{Neurorehabilitation protocol}

Intensive multifunctional neurorehabilitation was carefully individualized, calibrating the complexity of the exercises to the strength, endurance, range of motion, gait abnormalities, sensory deficits and related disability of each patient, as assessed individually. Over a three-week in-hospital period, neurorehabilitation was performed during twice a day, six days a week for at least two hours every session, grouping together HD patients who were hospitalized in the same period of time. Details of our protocol, including conventional neuromotor rehabilitation and occupational therapy (OT), were reported in a previous paper (Ciancarelli et al., 2013). The neuromotor protocol, according to current neurorehabilitation programs for HD patients (Busse et al., 2008), was aimed at improving balance and coordination, gait and posture, and muscle strength with respect to baseline performances, and at achieving maintenance of body control through specific exercises. A proprioceptive platform, the Biodex Balance System (Biodex Medical Systems Inc., Shirley, NY), was also utilized to improve static and dynamic postural stability on a static or unstable surface and to enhance kinesthetic abilities in patients with impaired proprioceptive reflex mechanisms. Light aerobic activities, such as cycling and walking, once a day, for no more than 20 minutes, were also included in the rehabilitation protocol. OT was planned to restore lost functional abilities in ADL, i.e. to recover global motor control, dexterity, and fine motricity. All the measured parameters were evaluated before and at the end of the three-week in-hospital rehabilitation treatment. The differences in the BI, TS and PPT scores recorded at baseline and at the end of the intervention were expressed as $\Delta$ scores. 


\section{Blood sampling}

Fasting venous blood samples were drawn from each study participant to obtain plasma or serum samples to be used for routine laboratory analyses and measurement of oxidative markers. Plasma and serum samples were frozen at $-80^{\circ} \mathrm{C}$ until analyses were performed. In the HD patients all the measured parameters were evaluated before and after rehabilitation.

\section{Laboratory analyses}

Among routine parameters, glucose, total cholesterol, and high-density lipoprotein cholesterol (HDL cholesterol) were analyzed using Architect c8000 (AbbottDiagnostic Clinical Chemistry Tests - Latina, Italy).

\section{Oxidative damage markers}

$\mathrm{Cu} / \mathrm{Zn}$-SOD serum levels $(\mathrm{ng} / \mathrm{ml})$ were measured using an ELISA kit (Bender MedSystems $\mathrm{GmbH}$, Vienna, Austria), with a sensitivity of $0.04 \mathrm{ng} / \mathrm{ml}$, an intra-assay coefficient of variation (CV) of $5.1 \%$, and an inter-assay CV of 5.8\%. Quantitative determination of serum NSE was detected by an ELISA kit (Alpha Diagnostic Intl Inc., San Antonio, TX, USA) with a sensitivity of $1.0 \mu \mathrm{g} / \mathrm{L}$, an intra-assay CV of $4-5 \%$ and an inter-assay CV of $10.3 \%$. Serum $8-O H d G$ was measured with a fast and sensitive competitive immunoassay (DNA Damage ELISA kit; Assay Designs, Ann Arbor, Michigan, USA) with an interassay CV of 6.4.

\section{Statistical analysis}

All data are given as means \pm standard error of the mean (S.E.M.). The non-parametric Mann-Whitney rank sum test was used to compare values obtained before and after neurorehabilitation in HD patients and in HD patients when compared to controls. The Wilcoxon matched-pairs signed rank test was used to compare mean $\mathrm{BI}$, TS and PPT scores before and after neurorehabilitation. Pearson's correlation analy-

Table I - Clinical characteristics of HD patients and controls. sis was performed to determine the relationship between variables of interest. Statistical significance was accepted if $p<0.05$. Statistical analyses were performed with the Statistical Package for Social Science (SPSS) for Windows, version 16.

\section{Results}

The clinical characteristics of the HD patients and controls are shown in table I. No significant differences in age, gender, glycemia, lipid profile or blood pressure were observed between the HD patients and the controls before the neurorehabilitation treatment. The BMI values of the HD patients were lower than those of the controls both before $(22.0 \pm 0.4$ vs $25.7 \pm 0.3, p<0.05)$ and after neurorehabilitation $(22.7 \pm 0.2$ vs $25.7 \pm 0.3$; $\mathrm{p}<0.05)$. No significant differences in BMI were observed in the HD patients, either the men or the women, when comparing the values recorded before and after neurorehabilitation $(22.0 \pm 0.4$ vs $22.7 \pm 0.2$; $\mathrm{p}=\mathrm{ns}$ ). Adherence to the neurorehabilitation protocol was evaluated daily by means of a checklist questionnaire. All the HD patients completed the intensive neurorehabilitation program with $100 \%$ overall compliance and without any adverse events. A significant improvement in clinical and functional outcome measures was observed at the end of the three-week treatment (Table II). No differences were found between men and women in the BI, TFCS, PPT, and TS scores, either before or after neurorehabilitation. As shown in figure 1 , serum levels of $\mathrm{Cu} / \mathrm{Zn}-\mathrm{SOD}(\mathrm{ng} / \mathrm{ml})$ and NSE $(\mu \mathrm{g} / \mathrm{ml})$ measured in the HD patients before neurorehabilitation were higher $(p<0.05)$ than those found in the controls (Cu/Zn-SOD: $51.61 \pm 4.20$ vs $30.06 \pm 3.32$; NSE: $15.83 \pm 0.92$ vs $9.66 \pm 3.32)$. The same values decreased significantly $(p<0.05)$ at the end of the neurorehabilitation program (Cu/Zn-SOD:41.62 \pm 2.82 vs $51.61 \pm 4.20, p<0.05$; NSE: $14.07 \pm 0.95$ vs $15.83 \pm 0.92$ ) but were still higher than those measured in the controls (Cu/Zn-SOD:41.62 \pm 2.82 vs $30.06 \pm 3.32$, $\mathrm{p}<0.05$; NSE: $14.07 \pm 0.95$ vs $9.66 \pm 3.32, p<0.05)$. As shown in figure 2, Cu/Zn-SOD and NSE correlated positively both before $(r=0.659 ; p=0.003)$ and after neurorehabilitation $(r=0.553, p=0.017)$. Serum values of $8-O H d G$ $(\mathrm{ng} / \mathrm{ml})$ were significantly higher $(\mathrm{p}<0.001)$ in the

\begin{tabular}{lll}
\hline & $\begin{array}{l}\text { HD patients } \\
(\mathrm{n}=18)\end{array}$ & $\begin{array}{l}\text { Controls } \\
(\mathrm{n}=10)\end{array}$ \\
\hline Gender $(\mathrm{n}$ men/women) & $13 / 5$ & $4 / 6$ \\
Age (years) & $51.11 \pm 2.77$ & $50.00 \pm 3.59$ \\
$\mathrm{BMI}\left(\mathrm{kg} / \mathrm{m}^{2}\right)$ & $22.0 \pm 0.4^{*}$ & $25.7 \pm 0.3$ \\
Systolic BP $(\mathrm{mmHg})$ & $140.57 \pm 5.22$ & $135.20 \pm 5.54$ \\
Diastolic BP $(\mathrm{mmHg})$ & $80.35 \pm 2.18$ & $76.40 \pm 2.30$ \\
Total cholesterol $(\mathrm{mg} / \mathrm{dl})$ & $170.0 \pm 4.7$ & $160.0 \pm 5.7$ \\
HDL-C $(\mathrm{mg} / \mathrm{dl})$ & $56.86 \pm 7.64$ & $54.60 \pm 4.23$ \\
Disease duration (age range, years) & $27-35$ & \\
Age at symptom onset (years) & $31.29 \pm 7.40$ & \\
TFCS (5 items) & $5.65 \pm 1.69$ & \\
\hline
\end{tabular}

Abbreviations: BMl=body mass index; HDL-C=high density lipoprotein cholesterol; TFCS=Total Functional Capacity Scale. Values are expressed as means \pm S.E.M. ${ }^{*} \mathrm{p}<0.05$ vs control values. 


\section{Ciancarelli et al.}

Table II - Outcome measures evaluated before and after intensive neurorehabilitation.

\begin{tabular}{llll}
\hline Scores & Before rehabilitation & After rehabilitation & $\Delta$ scores \\
\hline BI & $76.97 \pm 22.98$ & $85.88 \pm 18.32^{*}$ & $7.95 \pm 8.26$ \\
TS & $14.79 \pm 6.59$ & $19.44 \pm 6.63^{*}$ & $4.86 \pm 2.62$ \\
PPT & $21.91 \pm 7.21$ & $26.62 \pm 6.50^{*}$ & $4.50 \pm 2.69$ \\
TFCS & $5.65 \pm 1.69$ & $6.71 \pm 2.20^{*}$ & $1.09 \pm 0.61$ \\
\hline
\end{tabular}

Abbreviations: Bl=Barthel Index (10 items); TS=Tinetti Scale (16 items); TFCS=Total Functional Capacity Scale (5 items); PPT=Physical Performance test (9 items). $\Delta$ scores=differences between scores before and after rehabilitation. ${ }^{*} p<0.05$ after vs before scores.

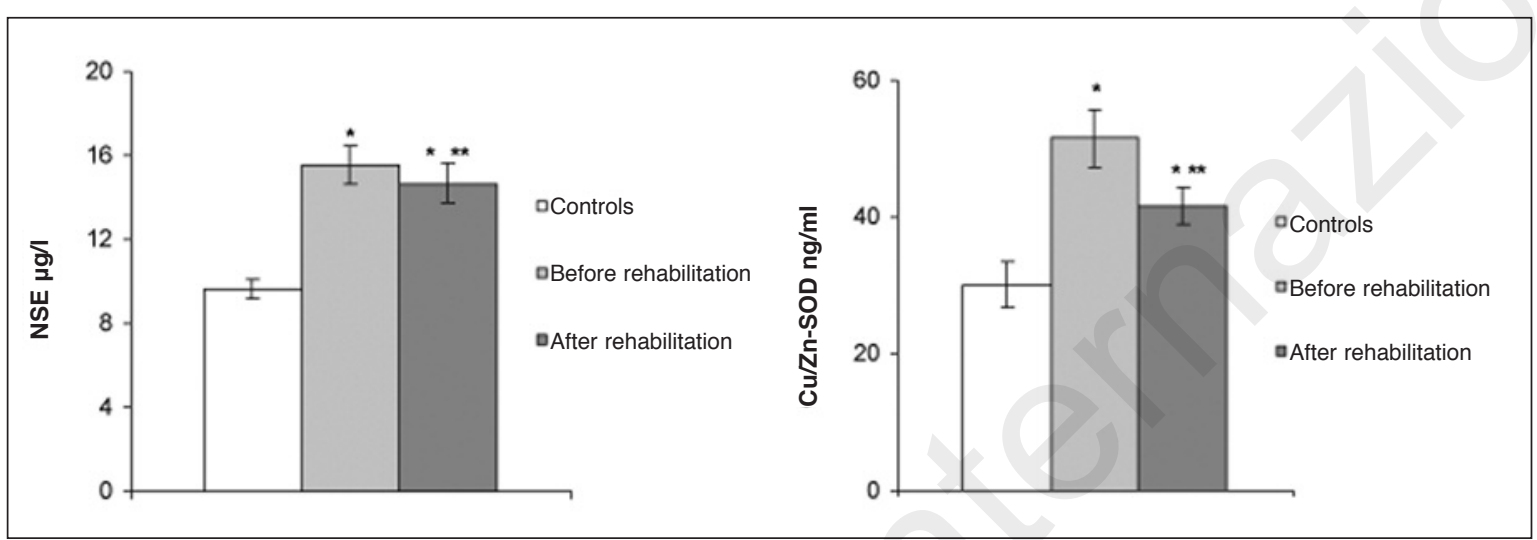

Figure 1 - Serum levels of NSE and Cu/Zn-SOD in controls and in HD patients before and after rehabilitation. Results are expressed as means \pm S.E.M. ${ }^{*} p<0.05$ vs controls; ${ }^{* *} p<0.05$ vs before rehabilitation.

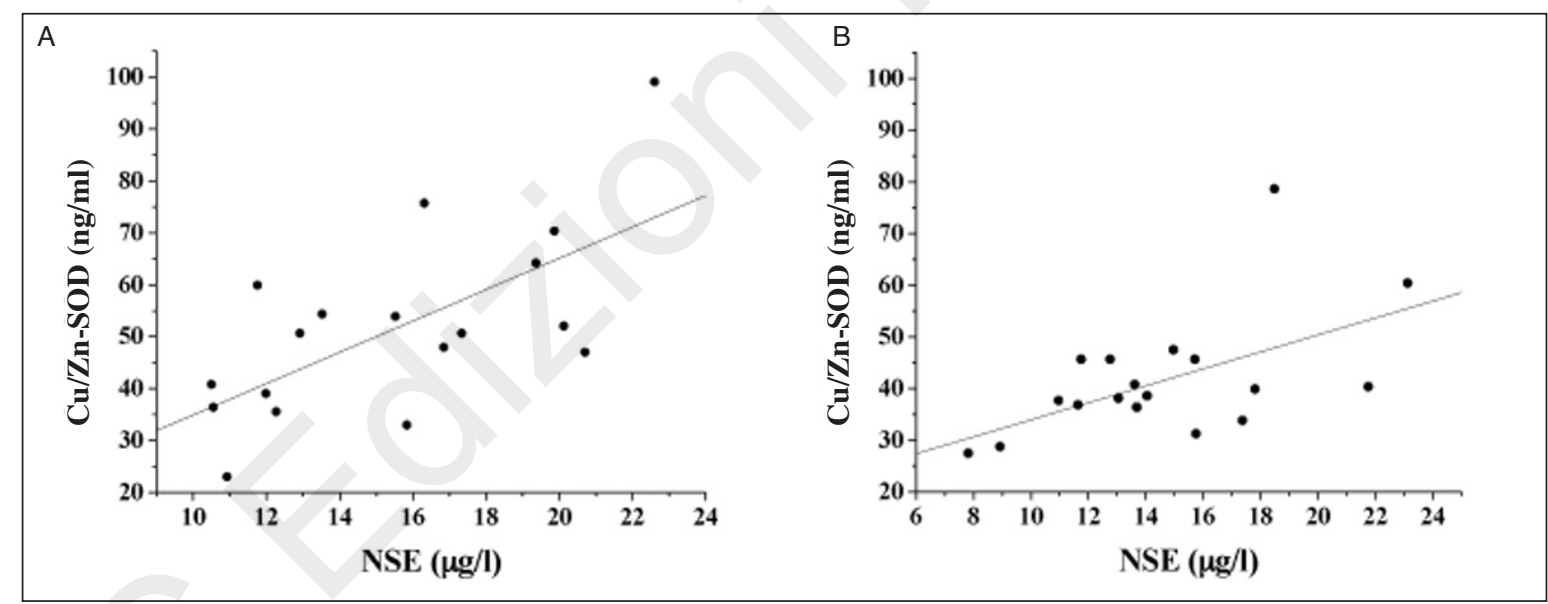

Figure 2 - Scatter plot of the relationship between Cu/Zn-SOD and NSE.

Cu/Zn-SOD and NSE correlated positively both before $(A)(p=0.002, r=0.659)$ and after $(B)(p=0.017, r=0.553)$ intensive neurorehabilitation.

patients before neurorehabilitation than in the controls $(60.98 \pm 12.78$ vs $3.61 \pm 0.54)$; these values decreased after neurorehabilitation, but the differences between the values detected before and after neurorehabilitation were not significant $(p=0.145)$. No gender-related differences were observed in the assessed oxidative markers either before or after neurorehabilitation.

\section{Discussion}

The findings of the present study confirm the occurrence of a significant redox imbalance detectable in the serum of symptomatic HD patients (Chen et al., 2007; Klepac et al., 2007; Hersch et al., 2006; Túnez et al., 2011), and, as a novel finding, provide evidence of the effectiveness of a three-week in-hospital intensive multifunctional neurorehabilitation program on modulation of serum levels of NSE, $8-O H d G$ and Cu/Zn-SOD, measured as potential peripheral markers of neuronal oxidative damage. Moreover, in accordance with previous studies (Greco et al., 2000; Miller et al., 2014), no gender-associated differences in the redox parameter values and functional outcome measures were detected, either before or after neurorehabilitation. Available studies suggest that neurons under various stress con- 
ditions release NSE into the systemic circulation, meaning that evaluation of its serum levels may potentially provide an indication of neuronal homeostatic alteration, as well as of neuron and glial cell loss (Killoran and Biglan, 2012; Shinozaki et al., 2009). Furthermore, it is widely accepted that serum levels of the metabolite $8-\mathrm{OHdG}$, an indicator of free radical damage of nucleic acids, is generally elevated in several neurodegenerative diseases in which oxidative damage has been implicated as a pathogenic mechanism (Hersch et al., 2006; Milakovic and Johnson, 2005; Stack et al., 2008). In line with these suggestions, the high serum levels of NSE and 8-OHdG which we found in HD patients before and after neurorehabilitation, might represent a peripheral expression of neuronal oxidative damage, and support the hypothesis that mhtt may be sufficient to induce a long-lasting excess of free radical formation resulting in diffusion of oxidative injury even outside the brain (Chen et al., 2007; Klepac et al., 2007; Zuccato et al., 2010). An efficient defence mechanism against superoxide radicals produced during mitochondrial respiration is provided by the activity of specific enzymes, superoxide dismutases, of which the Cu/Zn-SOD isozyme is the major SOD form in the vascular space. Because of its strategic location it plays a primary role as main enzymatic scavenger of superoxide anions, preventing or limiting the dangerous effects of superoxide anions at systemic levels (Di Massimo et al., 2006). The higher levels of Cu/Zn-SOD observed in HD patients, as compared with healthy age-matched subjects, may represent, in our opinion, a strategic compensatory mechanism that controls the systemic oxidative condition and the consequent cascade of oxidative events leading to the vascular alterations often observed in neurodegenerative diseases (Santamaria et al., 2001). The positive correlation that we observed between Cu/Zn-SOD and NSE before and after neurorehabilitation supports a neuroprotective role of $\mathrm{Cu} / \mathrm{Zn}-\mathrm{SOD}$ and it is likely that its persistently high level in HD patients could be elicited and sustained by the continuous free radical over-production due to mhtt-mediated mitochondrial dysfunction. There is evidence that HD patients potentially benefit from rehabilitation activities and that physical therapy is recommended for the treatment of HD (Arida et al., 2013; Bilney et al., 2003; Bohlen et al., 2013; Ciancarelli et al., 2013). Interestingly, findings in animal models showed that physical exercise is significantly involved in promoting cell proliferation and neuronal differentiation and that the exercise-mediated neuroplastic effects are probably related to a decreased expression of brain damage markers as well as to an increased bioavailability of a variety of neurotrophic factors (Al-Jarrah and Jamous, 2011; Tuon et al., 2012). Accordingly, the substantially decreased serum levels of NSE and 8-OHdG that we observed at the end of the rehabilitation program suggest that intensive multifunctional neurorehabilitation interferes with the redox equilibrium, thus potentially improving neuronal homeostasis. The lack of significant correlations between NSE, Cu/Zn-SOD and 8-
OHdG and functional evaluation tests observed both before and after neurorehabilitation, confirming previous results (Ciancarelli et al., 2014), provides evidence that these oxidative markers are inadequate or limited as potential peripheral indicators of functional autonomy, motor performances and physical abilities. Instead, their evaluation may be useful to better ascertain the contribution of oxidative stress either as a cause or as a consequence of the neuronal alteration and loss occurring in HD as well as to verify the effectiveness of therapeutic treatments, including rehabilitation therapy, aimed at decreasing oxidative stress. The results of the present study should be validated by an adequately powered multicenter case-control study conducted to investigate the effectiveness of this intensive neurorehabilitation on modulation of oxidative stress and its potential link with functional independence and motor performances in a larger sample of HD patients in the early and middle stages of the disease.

\section{References}

Al-Jarrah MD, Jamous M (2011). Effect of endurance exercise training on the expression of GFAP, S100B, and NSE in the striatum of chronic/progressive mouse model of Parkinson's disease. NeuroRehabilitation 28:359-363.

Andrew SE, Goldberg YP, Kremer B, et al (1993). The relationship between trinucleotide (CAG) repeat length and clinical features of Huntington's disease. Nat Genet 4:398-403.

Arida RM, Scorza FA, Cavalheiro EA (2013). Role of physical exercise as complementary treatment for epilepsy and other brain disorders. Curr Pharm Des 19:6720-6725.

Armstrong MJ, Miyasaki JM (2012). Evidence-based guideline: pharmacologic treatment of chorea in Huntington disease: report of the guideline development subcommittee of the American Academy of Neurology. Neurology 79:597-603.

Aziz NA, Jurgens CK, Landwehrmeyer GB, et al (2009). Normal and mutant HTT interact to affect clinical severity and progression in Huntington disease. Neurology 73:1280-1285.

Bilney B, Morris ME, Perry A (2003). Effectiveness of physiotherapy, occupational therapy and speech pathology for people with Huntington's disease: a systematic review. Neurorehabil Neural Repair 17:12-24.

Bohlen S, Ekwall C, Hellström K, et al (2013). Physical therapy in Huntington's disease-toward objective assessments? Eur J Neurol 20:389-393.

Borowsky B, Warner J, Leavitt BR, et al (2013). 8OHdG is not a biomarker for Huntington disease state or progression. Neurology 80:1934-1941.

Busse ME, Khalil H, Quinn L, et al (2008). Physical therapy intervention for people with Huntington disease. Phys Ther 88:820-831.

Camiletti-Moirón D, Aparicio VA, Aranda P, et al (2013). Does exercise reduce brain oxidative stress? A systematic review. Scand J Med Sci Sports 23:e202-212.

Chen CM, Wu YR, Cheng ML, et al (2007). Increased oxidative damage and mitochondrial abnormalities in the peripheral blood of Huntington's disease patients. Biochem Biophys Res Commun 359:335-340.

Ciancarelli I, De Amicis D, Di Massimo C, et al (2012). Oxidative stress in post-acute ischemic stroke patients after intensive neurorehabilitation. Curr Neurovasc Res 9:266-273.

Ciancarelli I, Tozzi Ciancarelli MG, Carolei A (2013). Effectiveness of intensive neurorehabilitation in patients 


\section{Ciancarelli et al.}

with Huntington's disease. Eur J Phys Rehabil Med 49:189-195.

Ciancarelli I, De Amicis D, Di Massimo C, et al (2014). Peripheral biomarkers of oxidative stress and their limited potential in evaluation of clinical features of Huntington's patients. Biomarkers 19:452-456.

Di Massimo C, Scarpelli P, Di Lorenzo N, et al (2006). Impaired plasma nitric oxide availability and extracellular superoxide dismutase activity in healthy humans with advancing age. Life Sci 78:1163-1167.

Eidelberg D, Surmeier DJ (2011). Brain networks in Huntington disease. J Clin Invest 121:484-492.

Greco A, Minghetti L, Levi G (2000). Isoprostanes, novel markers of oxidative injury, help understanding the pathogenesis of neurodegenerative diseases. Neurochem Res 25: 1357-1364.

Hersch SM, Gevorkian S, Marder K, et al (2006). Creatine in Huntington disease is safe, tolerable, bioavailable in brain and reduces serum $8 \mathrm{OH} 2$ 'dG. Neurology 66:250252.

Johri A, Beal MF (2012). Antioxidants in Huntington's disease. Biochim Biophys Acta 1822:664-674.

Killoran A, Biglan KM (2012). 8-OHdG: its (limited) potential as a biomarker for Huntington's disease. Biomark Med 6:777-780.

Klepac N, Relja M, Klepac R, et al (2007). Oxidative stress parameters in plasma of Huntington's disease patients, asymptomatic Huntington's disease gene carriers and healthy subjects: a cross-sectional study. J Neurol 254:1676-1683.

Mahoney FI, Barthel DW (1965). Functional evaluation: the Barthel Index. Md State Med J 14:61-65.

Milakovic T, Johnson GV (2005). Mitochondrial respiration and ATP production are significantly impaired in striatal cells expressing mutant huntingtin. J Biol Chem 280:30773-30782.

Miller E, Morel A, Saso L, et al (2014). Isoprostanes and neuroprostanes as biomarkers of oxidative stress in neurodegenerative diseases. Oxid Med Cell Longev 2014: 572491

Montoya A, Price BH, Menear M, et al (2006). Brain imaging and cognitive dysfunctions in Huntington's disease. J Psychiatry Neurosci 31:21-29.

Perluigi M, Poon HF, Maragos W, et al (2005). Proteomic analysis of protein expression and oxidative modification in R6/2 transgenic mice. Mol Cell Proteomics 4:1849-1861.

Reuben DB, Siu AL (1990). An objective measure of physical function in elderly outpatients. The Physical Performance Test. J Am Geriatr Soc 38:1105-1112.

Santamaría A, Pérez-Severiano F, Rodríguez-Martínez E, et al (2001). Comparative analysis of superoxide dismutase activity between acute pharmacological models and a transgenic mouse model of Huntington's disease. Neurochem Res 26:419-424.

Sassone J, Colciago C, Cislaghi G, et al (2009). Huntington's disease: the current state of research with peripheral tissues. Exp Neurol 219:385-397.

Shinozaki K, Oda S, Sadahiro T, et al (2009). S-100B and neuron-specific enolase as predictors of neurological outcome in patients after cardiac arrest and return of spontaneous circulation: a systematic review. Crit Care 13:R121.

Siesling S, van Vugt JP, Zwinderman KA, et al (1998). Unified Huntington's disease rating scale: a follow up. Mov Disord 13:915-919.

Shoulson I (1981). Huntington disease: functional capacities in patients treated with neuroleptic and antidepressant drugs. Neurology 31:1333-1335.

Sorolla MA, Reverter-Branchat G, Tamarit J, et al (2008). Proteomic and oxidative stress analysis in human brain samples of Huntington disease. Free Radic Biol Med 45:667-678.

Stack EC, Matson WR, Ferrante RJ (2008). Evidence of oxidant damage in Huntington's disease: translational strategies using antioxidants. Ann NY Acad Sci 1147:79-92.

Tinetti ME (1986). Performance-oriented assessment of mobility problems in elderly patients. J Am Geriatr Soc 34:119126.

Trushina E, McMurray CT (2007). Oxidative stress and mitochondrial dysfunction in neurodegenerative diseases. Neuroscience 145:1233-1248.

Tuon T, Valvassori SS, Lopes-Borges J, et al (2012). Physical training exerts neuroprotective effects in the regulation of neurochemical factors in an animal model of Parkinson's disease. Neuroscience 227:305- 312.

Túnez I, Sánchez-López F, Agüera E, et al (2011). Important role of oxidative stress biomarkers in Huntington's disease. J Med Chem 54:5602-5606.

Zielonka D, Marinus J, Roos RA, et al (2013). The influence of gender on phenotype and disease progression in patients with Huntington's disease. Parkinsonism Relat Disord 19:192-197.

Zuccato C, Valenza M, Cattaneo E (2010). Molecular mechanisms and potential therapeutical targets in Huntington's disease. Physiol Rev 90:905-981. 